TITLE:

\title{
Time-of-flight measurements of charge carrier diffusion in In_xGa_[1-x]N/GaN quantum wells
}

$\operatorname{AUTHOR}(S)$ :

Danhof, J.; Schwarz, U. T.; Kaneta, A.; Kawakami, Y.

\section{CITATION:}

Danhof, J....[et al]. Time-of-flight measurements of charge carrier diffusion in In_xGa_[1x]N/GaN quantum wells. PHYSICAL REVIEW B 2011, 84(3): 035324.

ISSUE DATE:

2011-07

URL:

http://hdl.handle.net/2433/161772

RIGHT:

(C2011 American Physical Society 
PHYSICAL REVIEW B 84, 035324 (2011)

\title{
Time-of-flight measurements of charge carrier diffusion in $\operatorname{In}_{x} \mathrm{Ga}_{1-x} \mathrm{~N} / \mathrm{GaN}$ quantum wells
}

\author{
J. Danhof* and U. T. Schwarz \\ Fraunhofer Institute for Applied Solid State Physics IAF, Tullastraße 72, D-79108 Freiburg, Germany and \\ Albert-Ludwigs-Universität Freiburg, IMTEK, Georges-Köhler-Allee 106, D-79110 Freiburg, Germany
}

A. Kaneta and Y. Kawakami

Kyoto University, Katsura Campus, Nishikyo-ku, Kyoto 615-2312, Japan

(Received 19 April 2011; revised manuscript received 10 May 2011; published 29 July 2011)

\begin{abstract}
Time-of-flight experiments were performed to investigate charge carrier diffusion in InGaN quantum wells. A mere optical setup with high spatial resolution was established on the basis of confocal microphotoluminescence microscopy in order to measure charge carrier movement directly. We investigate a multiquantum well sample emitting light at about $510 \mathrm{~nm}$ and found an ambipolar lateral diffusion constant of $0.25 \pm 0.05 \mathrm{~cm}^{2} / \mathrm{s}$.
\end{abstract}

DOI: 10.1103/PhysRevB.84.035324

PACS number(s): 73.50.Gr, 73.61.Ey, 78.47.da, 78.55.Bq

Indium gallium nitride ( $\mathrm{InGaN})$ is a material system that has proven to be very applicable for all kinds of semiconductor devices. Research has also found the system to be very interesting from a physical point of view. InGaN/GaN quantum wells (QWs) are known to suffer from a very large number of threading dislocations ${ }^{1,2}$ and material inhomogeneities ${ }^{3}$ due to growth on foreign substrates, strain, and solid phase immiscibility. ${ }^{4}$ The larger the indium content in the QW is the stronger the effects of these inhomogeneities become. ${ }^{5,6}$ Photoluminescence microscopy of such light emitting QWs show therefore fluctuations of intensity, energy, and full width at half maximum (FWHM) across the sample on different length scales. ${ }^{5,7}$ A lot of research has been dedicated to understand why QWs with such a low quality compared to, e.g., GaAs/AlGaAs QWs nevertheless emit light, even stimulated emission. Localization of charge carriers provides one explanation. ${ }^{8-10}$ Charge carriers are localized in minima of the energy landscape of the QW and thus prevented from traveling to nonradiative recombination centers. In this context, a carrier diffusion length of the order of tens of nanometers is expected in the low carrier density regime. Another possibility is that the charge carriers are prevented from reaching nonradiative recombination centers by potential barriers. ${ }^{11,12}$ We found direct evidence of lateral charge carrier transport in InGaN QWs. In this article, we describe these measurements with our time-of-flight setup.

On the basis of an idea by Hillmer et al. from more than 20 years ago, ${ }^{13}$ we created a setup for time-of-flight experiments that is precise enough to measure the small lateral diffusion coefficient in InGaN/GaN QWs. For this purpose, we modified a confocal microscope previously used for microphotoluminescence measurements (see Fig. 1). The spatial resolution of this confocal microscope is better than $400 \mathrm{~nm}$. The pinhole of the confocal setup, in our case a single mode optical fiber, was attached to a scanning stage to allow for $x-y$ scans of the microscope's image plane. We call this technique pinhole scan. ${ }^{14}$ This way, we can excite the sample by focusing a laser beam onto the sample, as one would for conventional confocal microscopy. By scanning the image plane, we can closely analyze variation of the sample's emission under inhomogeneous illumination, i.e., directly at the center of excitation by the laser or in its vicinity where less or no excitation from the laser takes place. The accuracy of our scanning stage is $10 \pm 20 \mathrm{~nm}$ which translates to $0.25 \pm 0.5 \mathrm{~nm}$ on the sample.

As a source of excitation, we used a picosecond pulsed frequency doubled Titan Sapphire laser with a pulse picker, set to a wavelength of $400 \mathrm{~nm}$. For nontime-resolved pinhole scans, the repetition rate of the laser was $80 \mathrm{MHz}$ and for detection, a $50 \mathrm{~cm}$ spectrometer with a liquid nitrogen cooled CCD chip was used. For time-of-flight experiments, temporal resolution has to be added. The repetition rate of the laser was set to $4 \mathrm{MHz}$ and a streak camera was used for time-resolved detection. The temporal resolution of the streak camera was 42 ps and the jitter was about 200 ps.

If excitation and detection happen at the same spot (confocal), the total carrier lifetime is being measured for samples with low charge carrier diffusion. If excitation and detection are spatially separated, one can observe a delayed onset of photoluminescence, corresponding to the time the carriers need to travel the distance between the locations of excitation and detection. The confocal technique suppresses scattered light. These time delays between the excitation of charge carriers and the detection of their emitted luminescence can be measured.

For our measurements, we use a green light emitting $c$-plane InGaN/GaN multi-QW (MQW) sample grown by Osram Opto Semiconductors. The MQW structure consists of seven $3 \mathrm{~nm}$ thick QWs with an indium content of about $26 \%$, separated by nominally undoped GaN barriers. The QWs are covered by a $10 \mathrm{~nm}$ thick GaN cap layer. The whole structure was grown on a GaN substrate to create a sample with a low threading dislocation density. ${ }^{15}$ The threading dislocation density is $5 \times$ $10^{7} \mathrm{~cm}^{-2}$. This low threading dislocation density allows us to find large enough dislocation free areas to perform our experiment.

Lateral transport of charge carriers can be described by a continuity equation: ${ }^{16}$

$$
\frac{\partial n}{\partial t}=G_{n}-\frac{n}{\tau_{n}}+D_{n} \Delta_{\perp} n,
$$

where diffusion is taken into account in the two-dimensional plane of the QW. $n$ represents the number of charge carriers and $G_{n}$ represents their generation rate. $\tau_{n}$ is the charge carrier 


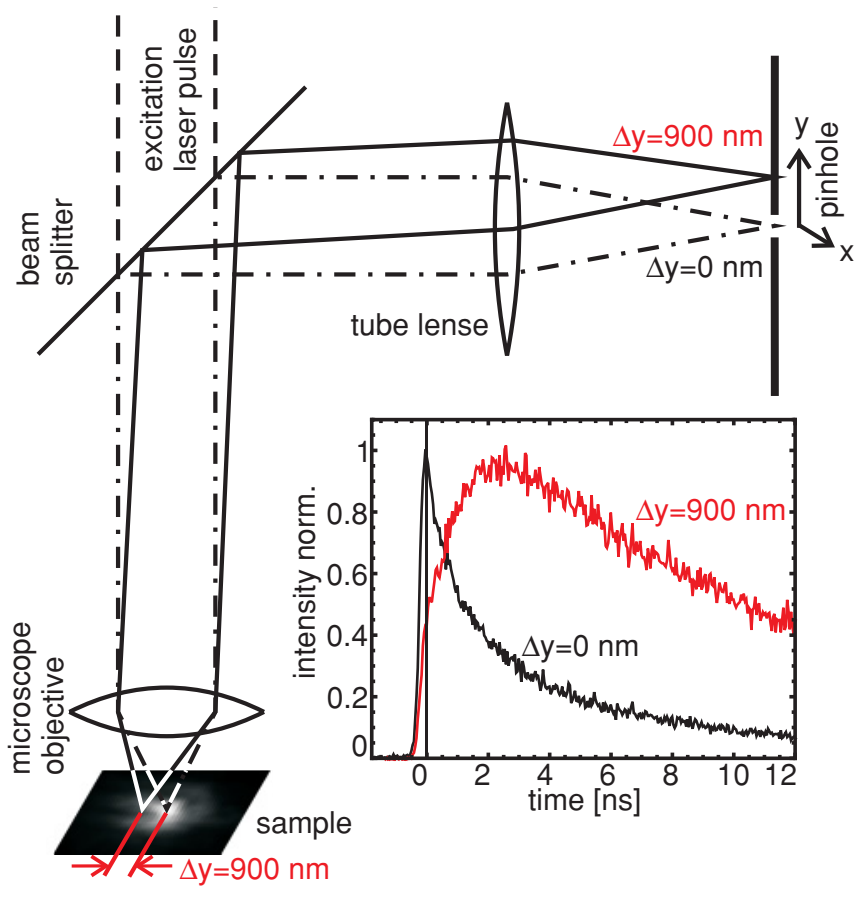

FIG. 1. (Color online) Experimental setup. The sample is excited by a laser beam focused with a microscope objective. In the pinhole plane, we observe a magnified image of the photoluminescence light from the sample. With a pinhole, we can thus select points of observation on the sample and analyze their temporal behavior with a streak camera. In the example, the distance between points of excitation and detection is $900 \mathrm{~nm}$.

life time and $D_{n}$ is the charge carrier diffusion constant. $\Delta_{\perp}$ is the 2D Laplace operator. In our case, the generation rate $G_{n}$ is zero after the excitation of the sample through a picosecond laser pulse. The differential equation is independent from the type of charge carriers that are observed and thus appropriate to describe ambipolar transport, i.e., the motion of an electronhole cloud interacting via Coulomb forces ${ }^{17}$ as well as the motion of excitons. This suits our experiment well as we can only observe recombining electrons and holes. There are indications that in a QW structure such as ours at room temperature excitons already dissociate into electron-hole pairs. ${ }^{18}$ With our experiment, we cannot distinguish between the two.

In this model, we are neglecting drift. When charge carriers are created in a $c$-plane InGaN/GaN QW structure, they reduce the quantum confined Stark effect (QCSE) by shielding the piezoelectric field which implicates a blueshift of the emission. The reduction of the QCSE together with the blueshift depends on the charge carrier density present in the QW. When we excite our sample locally, we therefore also induce a field within the plane of the QW which will cause drift overlaid with diffusion. The maximum of the electric field in the plane of the QW structure average over time was determined from the lateral energy shift to be $740 \pm 20 \mathrm{~V} / \mathrm{cm}$. Measurements with continuous wave excitation at the same sample suggest that at very high excitation densities, the internal lateral electric field might become as high as $1.4 \mathrm{kV} / \mathrm{cm}$. Drift caused by an accordant electric field can be integrated into the diffusion equation [Eq. (1)]. It was found that the field is not strong enough to take a significant influence on the motion of charge carriers in our sample. The effect of drift is thus neglected. However, the strong QCSE in green light emitting QWs creates long radiative charge carrier life times which is favorable for our experiment.

We describe the spatial distribution of the excitation density by the laser pulse on the sample by a Gaussian. Therefore, we also describe the created charge carrier density in the QW by a Gaussian, assuming that the dependence of the generation rate $G_{n}$ on the excitation density is an infinitely differentiable function. The shape of the charge carrier distribution in the sample at the time of excitation $(t=0 \mathrm{~ns})$ provides a boundary condition:

$$
n=e^{\left(-\frac{x^{2}+y^{2}}{2 r_{e}^{2}}\right)} .
$$

Parameters to be determined from the measurement are $\tau_{n}$, $r_{e}$, and $D_{n}$. The resulting differential equation can be solved numerically.

We excite our sample at a wavelength of $400 \mathrm{~nm}$. Charge carriers are only created resonantly in the MQW and not in the barriers. Therefore, all observed diffusion effects are due to charge carrier dynamics in the QW structure only. The assumption is made that all QWs contain the same charge carrier densities. ${ }^{19}$ We performed our measurements in the regime of high excitation density of the curve of the internal quantum efficiency (IQE) which is relevant for operating light emitting diodes and laser diodes. The actual charge carrier density in the sample at the time of excitation was estimated by measuring its IQE. An excitation density dependent IQE curve can be fitted with a third-order polynomial describing recombination mechanisms in $\mathrm{InGaN} / \mathrm{GaN}$ optical devices. This model is known as $\mathrm{ABC}$ model. ${ }^{20}$ From the fitting parameters A (Shockley-Read-Hall recombination, a nonradiative single-particle process), B (radiative recombination, a two-particle process), and C (Auger recombination, a threeparticle nonradiative process), the number of charge carriers in the QWs can be estimated. This is shown in Fig. 2. The peak charge carrier density in our measurement is roughly

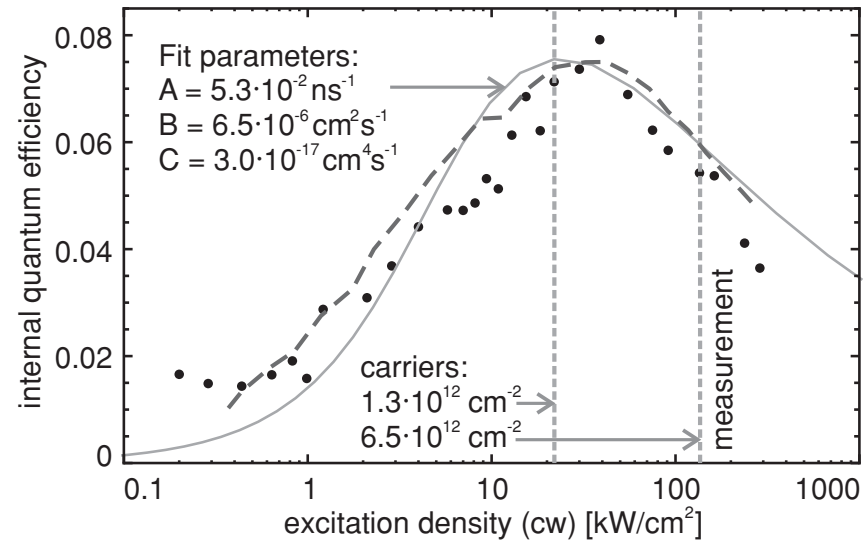

FIG. 2. Excitation dependent internal quantum efficiency. The gray dashed continuous line was determined from continuous wave excitation. The dots were derived from pulsed excitation. The gray continuous line resembles a fit with the fitting parameters given in the image. The peak charge carrier density in our experiment can thus be estimated to be about $6.5 \times 10^{12} \mathrm{~cm}^{-2}$. 


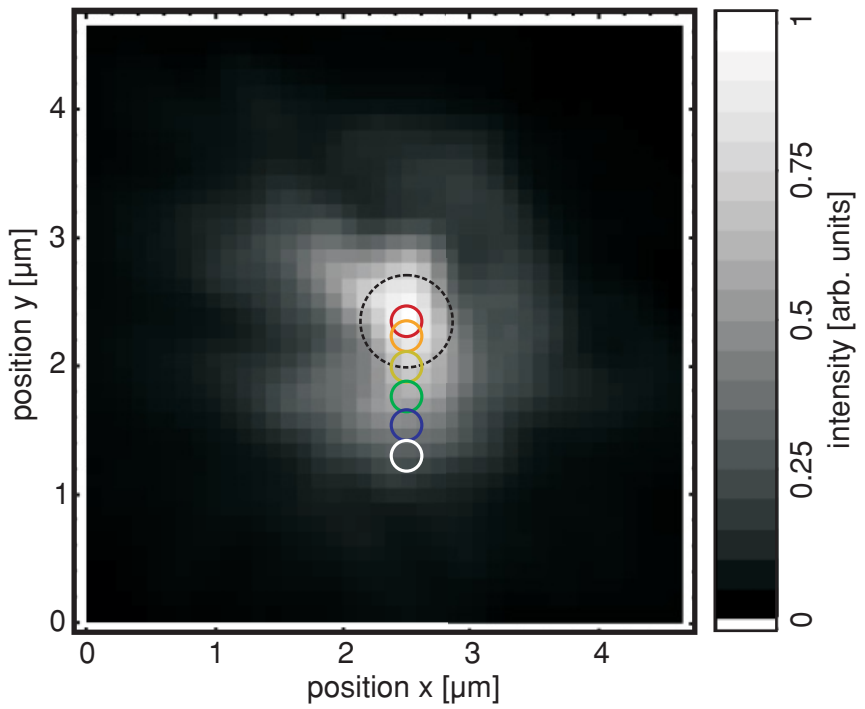

FIG. 3. (Color) Nontime-resolved pinhole scan of an InGaN MQW sample for orientation on the sample. The colored circles mark points where time-resolved measurements were taken. The dashes circle marks the FWHM of the laser spot at $\Delta y=360 \pm 10 \mathrm{~nm}$

$6.5 \times 10^{12} \mathrm{~cm}^{-2}$. Taking the Mott density to be of the order ${ }^{21}$ of $1 / \pi a_{0}^{2}$, with a Bohr radius $a_{0}$ of $2.5 \mathrm{~nm}$, we find that our charge carrier density is approximately the Mott density for bulk material.

We performed nontime-resolved pinhole scans in the immediate vicinity of the excitation spot to allow for orientation on the sample. The result is an intensity map as displayed in Fig. 3. The figure shows an image of the sample, the position of the exciting laser spot at the center. The bright area in the middle of the image caused by the exciting laser focus is not concentrical as would be expected. This is due to inhomogeneity of the sample already stated above. On this nontime-resolved scan, it is impossible to distinguish between charge carriers that recombine within the area that is hit by the laser and charge carriers that diffused away from the center of excitation and recombined there at a later time. For our measurements, we choose a spot within a particularly bright area to ensure a large signal-to-noise ratio and avoid threading dislocations and an unnecessary large number of point defects. Measurements within or in the vicinity of areas which appear dark in microphotoluminescence scans have also been performed. We observe a change in the signal's intensity rather than the diffusion constant.

To measure the charge carrier diffusion constant, a line scan from the center of excitation to the vicinity was performed. The scanning points for time-resolved pinhole scans are marked by colored circles in Fig. 3. Streak camera images of the actual time-resolved measurements are depicted in Fig. 4. Figure 4(a) was taken with matching excitation and detection position $(\Delta y=0)$. It shows directly the decay of intensity at the center of excitation after the laser pulse. At short wavelengths $(450-475 \mathrm{~nm})$, the signal decays very fast. The fact that this part of the emission is blueshifted very strongly and is only present at the highest excitation densities indicates that this is already stimulated emission from the QW. Analysis of InGaN laser diode performance just below lasing threshold has shown

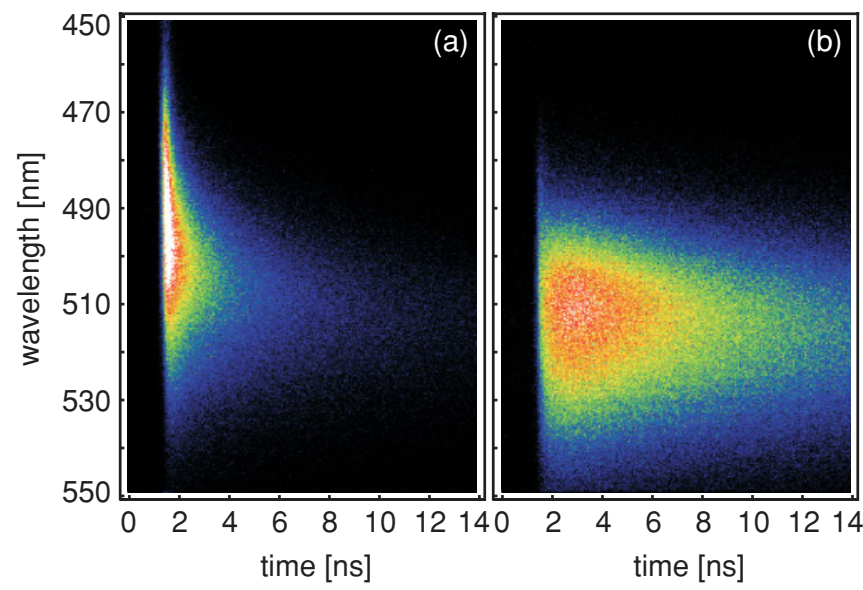

FIG. 4. (Color) Time-resolved spectra from time-resolved pinhole scans. (a) $\Delta y=0 \mathrm{~nm}$. Position of detection is the red circle in Fig. 3. (b) $\Delta y=675 \mathrm{~nm}$. Position of detection is the blue circle in Fig. 3. The color scaling was matched for good visible recognition. The maximum intensity of the measurement on the left side is a factor 14 larger than on the right side.

that there is already stimulated emission before actual lasing starts. $^{20,22}$ The shift in wavelength from $475 \mathrm{~nm}$ to longer wavelengths depending on the charge carrier density in the sample is typical for green light emitting InGaN/GaN QW samples and a consequence of the quantum-confined Stark effect.

Figure 4(b) depicts time-resolved spectra with a distance of $\Delta y=675 \mathrm{~nm}$ between the center of excitation and detection. This position of detection is marked by a blue circle in Fig. 3 . The emission peak intensity at this detection spot is about a factor 14 lower when compared to $\Delta y=0$. The emission wavelength of the sample is shifted to longer wavelengths here. This is again a result of the QCSE and the fact that here the charge carrier density is too low for stimulated emission. The very short pulse of emission at about $480 \mathrm{~nm}$ is due to scattered light. Most importantly, we observe a delay of the maximum of the emission intensity after the laser pulse. In Fig. 4(a), the maximum of the emission sets in directly at the laser pulse and from this point of time drops constantly. In Fig. 4(b), there is also emission immediately after excitation. However, the emission intensity $1 \mathrm{~ns}$ after the laser pulse is larger than directly at the time of excitation.

Time-resolved spectra like these were taken at different distances between excitation and detection (see the circles of different colors in Fig. 3). For quantitative analysis, one single decay line was calculated from the whole time-resolved plot by integrating from 522 to $534 \mathrm{~nm}$ within the whole time range. The result is shown in Fig. 5. We choose these long wavelengths to avoid any influence from scattered light or stimulated emission. A range of 522-534 nm gives us a good signal-to-noise ratio within the streak camera measurement while still avoiding disturbing effects. Other wavelength intervals show same results quantitatively.

The spectra in Fig. 5 can be divided into two groups. The first group with high intensity has its intensity maximum at $t=$ $0 \mathrm{~ns}$. From this point on, the intensity decays exponentially. All three spectra were taken within the FWHM of the excitation 


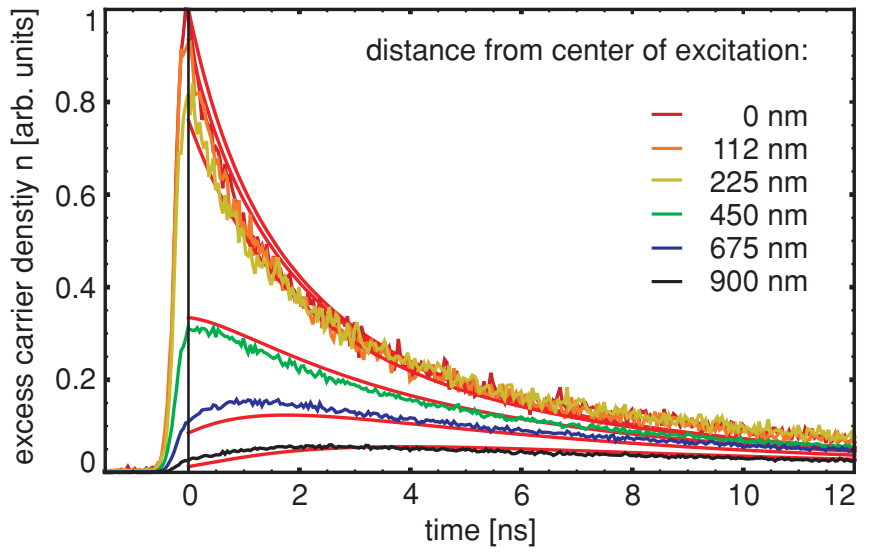

FIG. 5. (Color) Carrier density as a function of time at different radii. The intensity profile for different distances between excitation and detection over time is plotted. The measured curves are compared to the calculated curves (thick continuous lines).

laser spot. Within this area, obviously no charge carrier diffusion can be detected. The second group forms spectra at a distance of $450 \mathrm{~nm}$ between excitation and detection where the shape of the intensity decay over time begins to change.

These spectra can be used for a quantitative evaluation of the data. Under the assumption that the charge carrier diffusion constant is small, the charge carrier life time can be derived from the spectrum at $\Delta y=0$. Also, for the spectra that are relevant for the observation of diffusion ( $\Delta y=450 \mathrm{~nm}, \Delta y=$ $675 \mathrm{~nm}$, and $\Delta y=900 \mathrm{~nm}$ ), we find a single exponential decay of emission intensity with time. In case of this sample, the radiative charge carrier life time is $\tau_{n}=16 \pm 3 \mathrm{~ns}$. The other value that needs to be established is the FWHM of the excitation laser spot. $r_{e}$ can be determined from the intensities of the spectra at $t=0 \mathrm{~ns}$. This position is marked by a vertical line in Fig. 5. At $t=0 \mathrm{~ns}$, the light emitting area on the sample has not yet been broadened by diffusion. Since the spatial position with respect to the excitation center is known for all intensity decay curves in Fig. 5, the emission intensity versus position can be fitted by a Gaussian. The FWHM in case of this setup and this measurement is $720 \pm 20 \mathrm{~nm}$, i.e., $r_{e}=300 \pm 9 \mathrm{~nm}$. For illustration, a dashed line is shown in Fig. 3. The diameter of the circle around the center of excitation is the FWHM of the laser spot. Now all the parameters needed to solve Eq. (1) are known, except for the charge carrier diffusion constant $D_{n}$. This last parameter can be determined by variation. A value of $0.25 \pm 0.05 \mathrm{~cm}^{2} / \mathrm{s}$ gives, as solutions of Eq. (1), the continuous lines in Fig. 5. Deviations larger than $\pm 0.05 \mathrm{~cm}^{2} / \mathrm{s}$ are in conflict with our measured results and can be ruled out.

Table I shows an overview over diffusion constants from different materials and samples. All values listed below were determined by optical measurements. The diffusion constant of bulk GaAs was reported ${ }^{23}$ to be $20 \mathrm{~cm}^{2} / \mathrm{s}$. This is almost 2 orders of magnitude larger than our result. GaAs/AlGaAs quantum wells display even larger diffusion constants. ${ }^{13}$ Bulk $\mathrm{GaN}$ was found to have a charge carrier diffusion constant of $1.6-2.0 \mathrm{~cm}^{2} / \mathrm{s}$ depending on the material quality. ${ }^{24-26}$ This is about 1 order of magnitude larger than what we found for
TABLE I. Several literature values for diffusion constants of bulk GaAs, GaAs/AlGaAs quantum well, bulk GaN, and InGaN/GaN quantum well samples for comparison with our measurement results.

\begin{tabular}{lc}
\hline \hline Sample & Diffusion constant $\left(\mathrm{cm}^{2} / \mathrm{s}\right)$ \\
\hline Bulk GaAs $^{\mathrm{a}}$ & 20 \\
GaAs/AlGaAs quantum well $^{\mathrm{b}}$ & 56 \\
Bulk GaN $^{\mathrm{c}}$ & From 1.6 to 2.0 \\
$50 \mathrm{~nm} \mathrm{InGaN} \mathrm{layer}$ & 2.1 \\
InGaN/GaN quantum wells $^{\mathrm{e}}$ & From 0.51 to 0.62 \\
\hline
\end{tabular}

${ }^{\mathrm{a}}$ Reference 23 .

${ }^{\mathrm{b}}$ Reference 13 .

${ }^{\mathrm{c}}$ References 24-26.

${ }^{\mathrm{d}}$ Indium content $8 \%$, Reference 27.

${ }^{\mathrm{e}}$ Indium content between $22 \%$ and 40\%, Reference 28 .

our InGaN/GaN QW. A thick InGaN layer with an indium content of $8 \%$ was reported to show a diffusion constant of $2.1 \mathrm{~cm}^{2} / \mathrm{s}$, which is as large as the reported values for bulk $\mathrm{GaN}$. For InGaN/GaN QWs, there has been a report ${ }^{28}$ of diffusion constants from 0.51 to $0.62 \mathrm{~cm}^{2} / \mathrm{s}$ for an indium content between $22 \%$ and $40 \%$.

The significantly larger diffusion constants in GaAs/AlGaAs QWs compared to InGaN/GaN QWs accounts for the significantly lower homogeneity of InGaN QWs compared to GaAs/AlGaAs QWs. For GaAs/AlGaAs QWs, there have been reports about the influence of QW thickness on scattering mechanisms and thus charge carrier diffusion constants. For low temperatures $(40-100 \mathrm{~K})$ as well as thin QWs impurity-interface-roughness and barrier-alloy-disorder reduce the charge carrier diffusion. ${ }^{29}$ We expect these scattering mechanisms to have a much larger influence on charge carrier diffusions in InGaN/GaN MQWs. This is due to not only the large number of surfaces within a QW structure, but also the spontaneous and piezoelectric field, which was already mentioned above. Electrons and holes are separated by the electric field, and pushed toward the boundaries. There even random indium distribution of an otherwise perfectly grown QW provides roughness at which charge carriers are scattered. ${ }^{30,31}$

In conclusion, we performed time-of-flight measurements to investigate the ambipolar lateral diffusion constant in InGaN/GaN QWs. To do so, we established a setup for solely optical measurements. We reconfigurated a confocal microscope with high spatial resolution to allow for what we call pinhole scans: the pinhole which defines the area of detection scanned in the image plane; temporal resolution is achieved by combining a picosecond $\mathrm{Ti}$ : sapphire laser with a streak camera. With this setup, we directly observed charge carrier transport in a green light $(510 \mathrm{~nm})$ emitting InGaN/GaN MQW structure. The interpretation of our observations is confirmed by solving continuity and rate equation specifically for the measured sample. For the sample discussed in this work, we were able to determine a charge carrier diffusion constant of $0.25 \pm 0.05 \mathrm{~cm}^{2} / \mathrm{s}$.

The authors would like to thank Japan Society for the Promotion of Science (JSPS) and Deutsche Forschungsgesellschaft (DFG)(project SCHW 804/8-1) for funding. 
*julia.danhof@iaf-extern.fraunhofer.de

${ }^{1}$ N. Pauc, M. R. Phillips, V. Aimez, and D. Drouin, Appl. Phys. Lett. 89, 161905 (2006).

${ }^{2}$ J. C. Brooksby, J. Mei, and F. A. Ponce, Appl. Phys. Lett. 90, 231901 (2007).

${ }^{3}$ T. B. Bartel, C. Kisielowski, P. Specht, T. V. Shubina, V. N. Jmerik, and S. V. Ivanov, Appl. Phys. Lett. 91, 101908 (2007).

${ }^{4}$ I. H. Ho and G. B. Stringfellow, Appl. Phys. Lett. 69, 2701 (1996).

${ }^{5}$ B. Han, B. W. Wessels, and M. P. Ulmer, J. Appl. Phys. 99, 084312 (2006).

${ }^{6}$ A. Kaneta, M. Funato, and Y. Kawakami, Phys. Rev. B 78, 125317 (2008).

${ }^{7}$ H. Masui, T. Melo, J. Sonoda, C. Weisbuch, S. Nakamura, and S. P. DenBaars, J. Electron. Mater. 39, 15 (2010).

${ }^{8}$ S. Chichibu, T. Azuhata, T. Sota, and S. Nakamura, Appl. Phys. Lett. 69, 4188 (1996).

${ }^{9}$ Y. Narukawa, Y. Kawakami, M. Funato, S. Fujita, S. Fujita, and S. Nakamura, Appl. Phys. Lett. 70, 981 (1997).

${ }^{10}$ J. S. Im, S. Heppel, H. Kollmer, A. Sohmer, J. Off, F. Scholz, and A. Hangleiter, J. Cryst. Growth 189/190, 597 (1998).

${ }^{11}$ S. Sonderegger, E. Feltin, M. Merano, A. Crottini, J. F. Carlin, B. Deveaud, N. Grandjean, and J. D. Ganière, Appl. Phys. Lett. 89, 232109 (2006).

${ }^{12}$ N. K. van der Laak, R. A. Oliver, M. J. Kappers, and C. J. Humphreys, Appl. Phys. Lett. 90, 121911 (2007).

${ }^{13}$ H. Hillmer, A. Forchel, S. Hansmann, E. Lopez, and G. Weimann, Solid-State Electron. 31, 485 (1988).

${ }^{14}$ C. Vierheilig, H. Braun, U. T. Schwarz, W. Wegscheider, E. Baur, U. Strauß, and V. Härle, Phys. Status Solidi C 4, 2362 (2007).

${ }^{15}$ J. Danhof, C. Vierheilig, U. T. Schwarz, T. Meyer, M. Peter, B. Hahn, M. Meier, and J. Wagner, Phys. Status Solidi C 6, 747 (2009).

${ }^{16}$ S. L. Chuang, Physics of Optoelectronic Devices (Wiley, New York, 2009).
${ }^{17}$ H. Hillmer, S. Hansmann, A. Forchel, M. Morohashi, E. Lopez, H. P. Meier, and K. Ploog, Appl. Phys. Lett. 53, 1937 (1988).

${ }^{18}$ C. Netzel, V. Hoffmann, T. Wernicke, A. Knauer, M. Weyers, M. Kneissl, and N. Szabo, J. Appl. Phys. 107, 033510 (2010).

${ }^{19}$ D. S. Sizov, R. Bhat, A. Zakharian, J. Napierala, K. Song, D. Allen, and C. Zah, Appl. Phys. Express 3, 122101 (2010).

${ }^{20}$ U. T. Schwarz, Proc. SPIE 7216, 72161 U (2009).

${ }^{21}$ C.-K. Sun, S. Keller, G. Wang, M. S. Minsky, J. E. Bowers, and S. P. DenBaars, Appl. Phys. Lett. 69, 1936 (1996).

${ }^{22}$ S. Grzanka, P. Perlin, R. Czernecki, L. Marona, M. Boćkowski, B. Łucznik, M. Leszczyński, and T. Suski, Appl. Phys. Lett. 95, 071108 (2009).

${ }^{23}$ B. A. Ruzicka, L. K. Werake, H. Samassekou, and H. Zhao, Appl. Phys. Lett. 97, 262119 (2010).

${ }^{24}$ T. Malinauskas, K. Jarašiūnas, S. Miasojedovas, S. Juršènas, B. Beaumont, and P. Gibart, Appl. Phys. Lett. 88, 202109 (2006).

${ }^{25}$ S. M. Olaizola, W. H. Fan, S. A. Hashemizadeh, J.-P. R. Wells, D. J. Mowbray, M. S. Skolnick, A. M. Fox, and P. J. Parbrook, Appl. Phys. Lett. 89, 072107 (2006).

${ }^{26}$ S. Juršènas, S. Miasojedovas, A. Žukauskas, B. Łucznik, I. Grzegory, and T. Suski, Appl. Phys. Lett. 89, 172119 (2006).

${ }^{27}$ R. Aleksiejūnas, M. Sūdžius, T. Malinauskas, K. Jarašiūnas, Q. Fareed, G. Gaska, M. S. Shur, J. Zhang, J. Yang, E. Kuokštis, and M. A. Khan, Phys. Status Solidi C 7, 2686 (2003).

${ }^{28}$ K. Okamoto, A. Kaneta, K. Inoue, Y. Kawakami, M. Terazima, G. Shinomiya, T. Mukai, and Sg. Fujita, Phys. Status Solidi B 228, 81 (2001).

${ }^{29}$ H. Hillmer, A. Forchel, and C. W. Tu, J. Phys. Condens. Matter 5, 5563 (1993).

${ }^{30}$ M. Gallart, Y. Morel, T. Taliercio, P. Lefebvre, B. Gil, J. Allègre, H. Mathieu, N. Grandjean, M. Leroux, and J. Massies, Phys. Status Solidi A 180, 127 (2000).

${ }^{31}$ D. Watson-Parris, M. J. Godfrey, P. Dawson, R. A. Oliver, M. J. Galtrey, M. J. Kappers, and C. J. Humphreys, Phys. Rev. B 83, 115321 (2011). 